\title{
Frequency-dependent viabilities of Drosophila pseudoobscura karyotypes
}

\author{
Wyatt W. Anderson, Jonathan Arnold, \\ Scott A. Sammons \\ and Darrell G. Yardley*
}

\begin{abstract}
Department of Genetics, University of Georgia, Athens, Georgia 30602, U.S.A.

* Department of Zoology, Clemson University, Clemson, South Carolina 29631, U.S.A.
\end{abstract}

The viabilities between egg and adult life stages of Drosophila pseudoobscura karyotypes were studied at low, intermediate, and high frequencies. The viabilities of pairs of karyotypes were compared at each frequency and the viabilities of the three karyotypes, at one combination of frequencies. Eggs were counted into vials and samples taken of the adults emerging after viability selection. ST and $\mathrm{CH}$ gene arrangements of the third chromosome carrying different amylase alleles were used, and the karyotypes of adult flies were scored by gel electrophoresis. A statistical method related to the loglinear model was developed for estimating viabilities. This method takes account of the additional variability between replicates common in experiments of this kind and allows testing of nested hypotheses about the mechanism of selection. The viabilities of the homokaryotypes relative to the heterokaryotype were significantly higher at the low homokaryotypic frequency than at the higher ones. These viabilities do not show a consistent heterozygote advantage. This pattern of frequency-dependent viabilities will lead to a protected polymorphism for the gene arrangements, even in the absence of heterozygote advantage.

\section{INTRODUCTION}

The last 20 years have witnessed a major change in our views on the maintenance of genetic polymorphisms. Until the middle 1960s, heterozygote fitness advantage was believed to be a major force keeping alleles in balanced polymorphism - that is, at frequencies too high to be explained by recurrent mutation alone. The idea that heterozygote advantage could produce genetic polymorphisms originated in the early mathematical theories of population genetics, appearing first in Fisher (1922), and later in Haldane (1924) and Wright (1931). Th. Dobzhansky in particular emphasised its importance, and his views had a strong influence on the developing field of population genetics. $\mathrm{He}$ believed that heterozygote advantage was the simplest, and hence most acceptable, explanation for the selection he observed in natural and laboratory populations of Drosophila (Dobzhansky, $1947 a ; 1951$ ).

In the 1950s, evidence began to accumulate that other forms of selection not necessarily requiring heterozygote advantage could produce balanced polymorphisms. Levene (1953) showed that habitat diversity, with selection differing among parts of a population, could maintain a balanced polymorphism without heterozygote advantage in every habitat. The interaction of genotypes in determining each other's fitness (Levene et al., 1954) and the effects on fitness of factors such as larval density (Lewontin, 1955) were demonstrated. Nevertheless, heterozygote advantage remained in the eyes of most geneticists the major cause of balanced polymorphisms.

In the 1960s a flurry of experimental studies began to provide examples of selection which depended on genotypic frequency. Examples include the mating advantage of rare males (Ehrman et al., 1965; Petit and Ehrman, 1969), larval conditioning of Drosophila food medium (Dawood and Strickberger, 1969), and formation of predator search images (the apostatic selection of Clarke, 1962). It became clear that genetic polymorphisms could be maintained in several ways, and that heterozygote advantage was only one of them (see, e.g., Dobzhansky, 1970, and Dobzhansky et al., 1977). This viewpoint is the prevalent one today.

The inversion polymorphism of $D$. pseudoobscura is a classic instance of balancing selection, which is a convenient term for the whole complex of selective processes producing balanced polymorphisms. A series of gene arrangements on the 
third chromosomes, formed by overlapping inversions, segregate as if they were alleles at a single locus. The inversions involve a sizable part of the genome, and the contents of the inversions are kept intact by the suppression of recombination in heterozygotes. Thus, it is proper to speak of this system as a supergene. Natural selection is known to regulate the frequencies of these inversions in nature (Dobzhansky, 1943; 1944; Anderson et al., 1979; Salceda and Anderson, 1985) and in the laboratory (Wright and Dobzhansky, 1946; Anderson et al., 1968). For many years the selection on these arrangements was attributed largely to heterozygote advantage. Indeed, frequency changes in experimental populations often accord well with a model of constant selection and heterozygote advantage; they are the basis of the typical "selection curves" in a number of textbooks of genetics and evolution. As evidence accumulated that selection varies with karyotypic frequency, density, and other factors such as temperature, this view changed to one of balancing selection in which heterozygote advantage plays a major role. Today the role of heterozygote advantage in selection on $D$. pseudoobscura inversions is not clear.

Only a few analyses of components of selection in D. pseudoobscura have been carried out, because the gene arrangements can be scored in adults only through a laborious series of crosses (Arnold, 1981, and Arnold and Morrison, 1985). The fertility component of fitness, which is largely associated with female fecundity and male mating success, was estimated within single generations by Anderson and Watanabe (1974), who found large fertility differences between a heterokaryotype and the two corresponding homokaryotypes. In their experiments both male and female fertilities varied with karyotype frequency. Anderson and Brown (1984) found a rare male mating advantage of the D. pseudoobscura homokaryotypes relative to the heterokaryotype, although the heterokaryotypes did not show a rare male mating advantage.

There are no comparable measures of viability, a major component of fitness, at different inversion frequencies. The only direct study of karyotypic viabilities was made by Dobzhansky $(1947 b)$. He reared egg samples from a polymorphic cage population in uncrowded conditions, with extra yeast added to the cultures every few days. He compared karyotypic frequencies under these nearly-optimal conditions with Hardy-Weinberg expectations. In a similar way, he studied the karyotypic frequencies of males and females emerging from food cups and those of older males from the cage and concluded that significant viability selection occur- red between egg and adult life stages, and between newly-emerged and older males.

Our experiments were set up to test for a frequency-dependence of viability selection comparable to that of fertility selection (Anderson and Watanabe, 1974). We also present a new statistical methodology for viability selection which is related to the loglinear model of discrete multivariate statistics (Fienberg, 1980).

\section{MATERIALS AND METHODS}

Strains of D.pseudoobscura homokaryotypic for Standard (ST) and Chiricahua $(\mathrm{CH})$ gene arrangements on the third chromosome were utilised; they were isolated from a collection at Mather, California, in 1959 and carry the inversions on a genetic background from Mather. The $\alpha$-amylase locus is located within the inverted regions which differentiate $\mathrm{CH}$ and ST, and thus the $A m y^{0.84}$ and $A m y^{1 \cdot 00}$ alleles will remain associated with $\mathrm{CH}$ and ST gene arrangements and can be used as markers for the gene arrangements in adult flies. By pair matings when necessary, we derived five strains of ST fixed for $A m y^{1.00}$, and five of $\mathrm{CH}$ fixed for $A m y^{0.84}$. Crosses among the strains were arranged to produce homokaryotypic and heterokaryotypic eggs. The lines were intercrossed within ST and $\mathrm{CH}$ strains to generate homokaryotypes, and between the two types to generate heterokaryotypes, so that no egg used in the experiment was homokaryotypic for any one chromosome from nature.

Inseminated females were placed in egg-laying chambers consisting of glass dishes topped by plastic chimneys and plugged with cotton. Each dish contained food medium to which bone black was added to facilitate counting of eggs. Our food contained agar, cornmeal, molasses, brewer's yeast, and propionic acid (to inhibit mold). A solution of active yeast was painted on the surface of the food to stimulate egg-laying and to feed the laying females. Eggs produced by each female on the first day of laying were discarded as some might be infertile. Eggs of the three karyotypes were counted out and placed in plastic vials containing about $15 \mathrm{ml}$ of food medium. They were reared in incubators at $25 \pm 1^{\circ} \mathrm{C}$ and $60-70$ per cent relative humidity. One hundred eggs laid over a 10 -hour period were put into each vial. A preliminary experiment with densities of 50,100 , and 200 eggs showed that about half the eggs survived to become adults at the density of 100 , and that differences between the karyotypes would be revealed under this degree of crowding. 
The karyotypes were combined by pairs at three frequencies and in a triple combination at one frequency (table 1), and we studied at least 10 replicate vials of each combination. The first adults appeared on the thirteenth or fourteenth day, and most emerged within a five day period. Adults were removed after the first week of emergence and frozen at $-20^{\circ} \mathrm{C}$; a second collection was made at the end of the second week. Acrylamide gel electrophoresis and staining for amylase were performed as described by Yardley et al. (1977). In vials with only a pair of karyotypes, 10 females and 10 males were sampled from each vial when possible; occasionally 10 males or females were not available, or a gel was unscorable. In vials with three karyotypes, all surviving adults were karyotyped.

Density, karyotypic frequencies, replicate, and sex were all factors controlled in these experiments. We are particularly interested in the conditions which determine what Prout (1968) has called a protected polymorphism; that is, conditions which bring about the increase of either allele when its frequency is close to 0 . These conditions guarantee that both alleles remain in the population, although the position of any interior equilibrium is not specified. The conditions for a protected polymorphism are determined near gene frequencies of 0 and 1 , where one homozygote or the other will have a frequency close to 0 . Thus, only two genotypes are involved in testing for a protected polymorphism. Our experiments were designed to test for a protected polymorphism of the ST and $\mathrm{CH}$ gene arrangements, and we accordingly examined mixtures of karyotypes in pairwise comparisons. Other experimenters (Prout, 1971; Yoshimaru and Mukai, 1979) have used this design, which is also recommended on statistical grounds (see Cornell, 1973; 1979). To measure the three-way interaction between karyotypes, we also examined one combination with all three karyotypes present. These 10 combinations of karyotypic frequencies allowed us to study the response of homokaryotype fitness to variation in the input ratios of the karyotypes, as shown in fig. 1 . The points in the triangular plot (fig. 1) represent the mixtures at which viabilities were estimated.

\section{STATISTICAL ANALYSIS OF VIABILITY}

A common problem in analysing data from laboratory experiments is that the counts of karyotypes on which estimates of viability are based are more variable than those expected under binomial sampling (see Prout, 1971, and Clark and Doane, 1984). We have used a close relative of the loglinear model (Fienberg, 1980) to accommodate excess variability among replicates. This model leads to estimates of viability identical to those used by geneticists for many years (Haldane, 1956), as displayed in equation (2) below, but it rescales variances to reflect variability among replicates. The additional variability in viability estimates is generated by allowing survival probabilities from egg to adult stage to vary randomly from vial to vial.

In our mixture experiments each vial initially contained 100 eggs in two or three karyotypic classes in the ratio $3: 1 ; 1: 1,1: 3$, or $1: 2: 1$. One to three days after the emergence of adults, 10 adult flies of each sex were usually sampled and karyotyped using the electrophoretic markers $A m y^{0.84}$ and $A m y^{1 \cdot 00}$ for $\mathrm{CH}$ and ST, respectively. Since substantial fitness interactions between karyotypes were demonstrated previously (e.g., Levene et al., 1954), we analysed each pairwise competition experiment separately.

Our experiment to compare the viabilities of two karyotypes is outlined below for the ST/CH and $\mathrm{CH} / \mathrm{CH}$ karyotypes.

Table 1 Percentage frequencies of Drosophila pseudoobscura karyotypes among eggs before viability selection and among adults afterwards, and estimates of the relative viabilities of the karyotypes. $N_{R}$ is the number of replicate cultures, each begun with $100 \mathrm{eggs} ; N_{A}$ is the number of adults analysed; and S.E. is the standard error of a viability estimate.

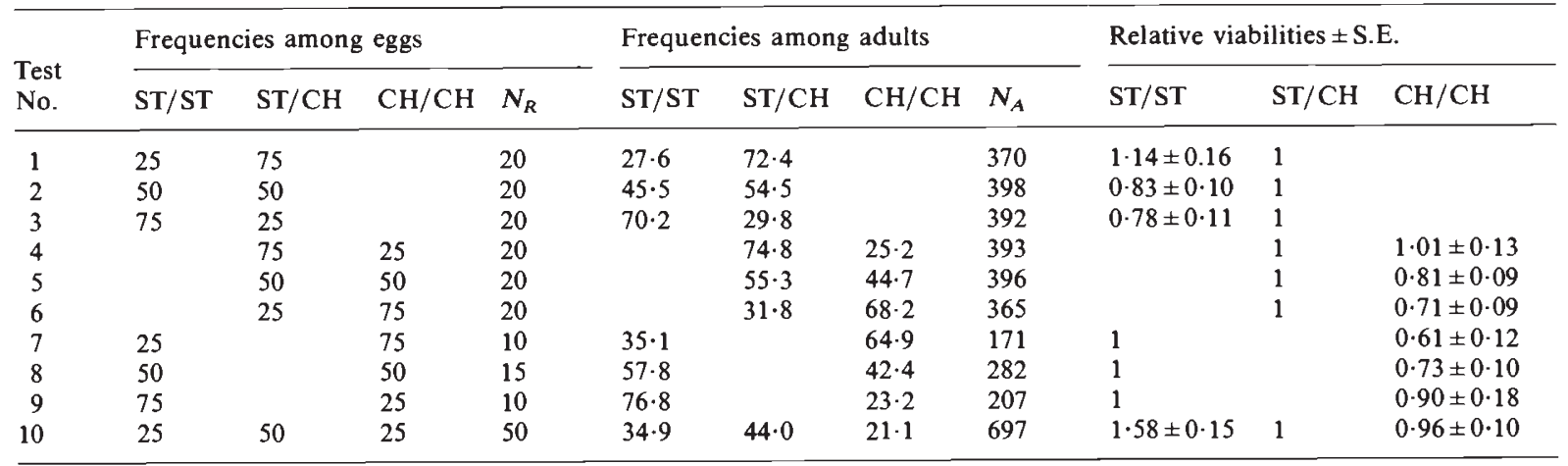



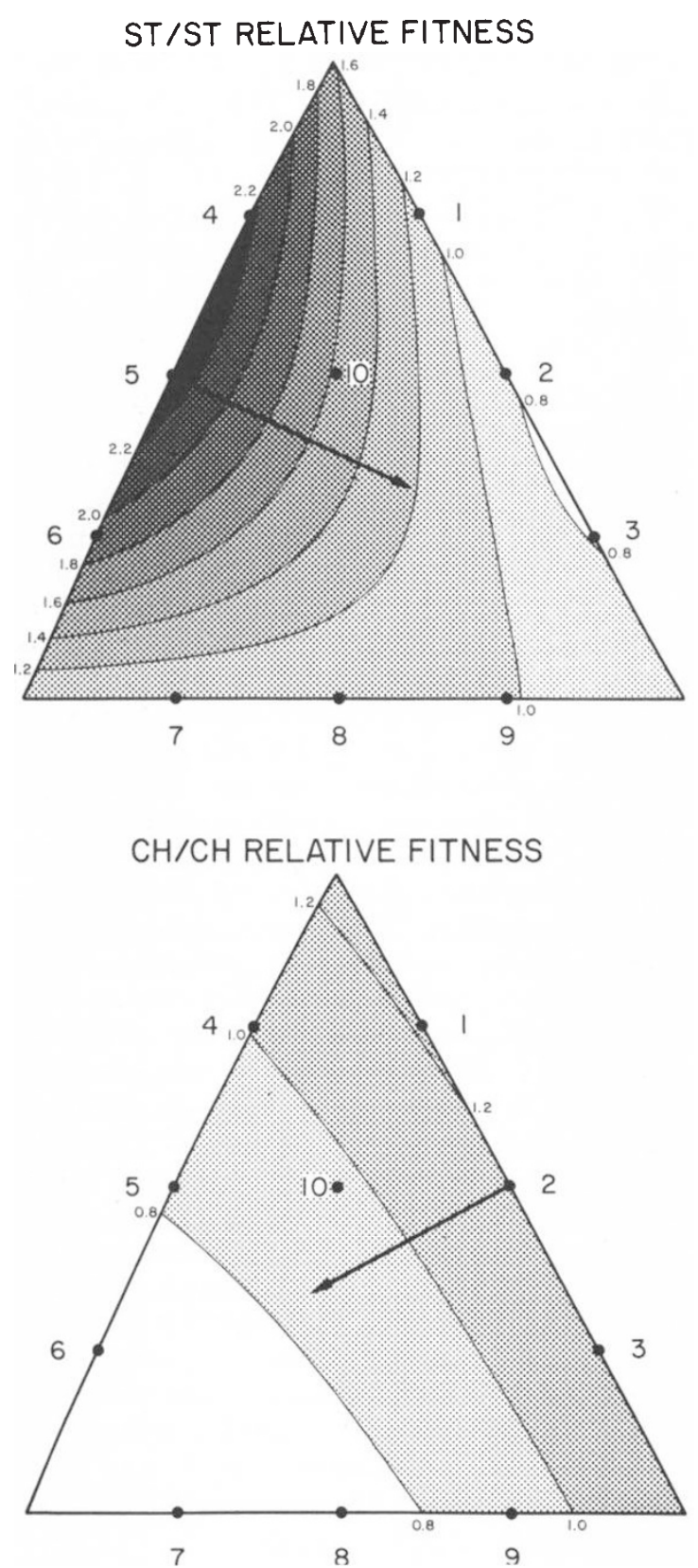

Figure 1 A triangular plot of mixture combinations in each experiment, on which is superimposed a contour plot of relative homokaryotypic fitness. The perpendicular distance from a point to a side represents the input frequency of a karyotype: from the base, ST/CH; from the left side, ST/ST; and, from the right side, $\mathrm{CH} / \mathrm{CH}$. The 10 points represent the 10 mixtures of karyotypes in table 1 at which relative fitness was estimated. Contours with their levels indicated by a decimal point are calculated by linear interpolation of relative homokaryotypic fitness between input frequencies. The arrows indicate the directions in which homokaryotypic frequencies (upper, ST/ST; lower, $\mathrm{CH} / \mathrm{CH}$ ) increase.
Karyotype

Input Ratio

Viability

Expected Probability

of Survival

Adult Numbers

$$
\begin{array}{cc}
\mathrm{ST} / \mathrm{CH} & \mathrm{CH} / \mathrm{CH} \\
k & 1 \\
1 & V_{k} \\
\pi_{k}=\begin{array}{c}
k /\left(k+V_{k}\right) \\
n_{1}
\end{array} & \bar{\pi}_{k}=V_{k} /\left(k+V_{k}\right) \\
n_{2}
\end{array}
$$

Males and females were counted separately. We refer to the individuals of a single sex within one replicate culture as a sample. Each sample yields $n_{1}$ flies of karyotype ST/CH, and $n_{2}$ of $\mathrm{CH} / \mathrm{CH}$. The sample size $n=n_{1}+n_{2}$ is usually 10 . Conditional on the probability of survival $\theta_{k}$, the count $n_{1}$ is binomial with parameters $\theta_{k}$ and $n$. When the probability of survival from egg to adult, $\theta_{k}$, is "fixed" as opposed to "random", then Haldane (1956) showed that a nearly unbiased estimator of the viability $V_{k}$ of $\mathrm{CH} / \mathrm{CH}$ relative to $\mathrm{ST} / \mathrm{CH}$ is $\hat{V}_{k}=k n_{2} /\left(n_{1}+1\right)$, the maximum likelihood estimator corrected for bias. The variance of this viability estimate, $\operatorname{VAR}\left(\hat{V}_{k}\right)=$ $V_{k}\left(k+V_{k}\right)^{2} /(k n)$, can be used to assess the variability between samples. The variability in our viability estimates over replicate vials does exceed that expected on the basis of binomial sampling, as shown in table 2 (e.g., $\left.\chi^{2}=64 \cdot 69, \mathrm{df}=37\right)$.

One possible explanation for this excess variability is that the probability of survival $\theta_{k}$ has a random component due to small microenvironmental differences between vials, and it seems reasonable to assume that $\theta_{k}$ has a beta distribution with parameters $n_{e} \pi_{k}$ and $n_{e} \bar{\pi}_{k}$, with $\bar{\pi}_{k}=1-\pi_{k}$. The parameters in the model are the expected probabilities of survival over replicates and the effective population size $n_{e}$ in a vial. The expected survival probabilities are considered to have some loglinear modelling structure on them (Fienberg, 1980), and the effective population size in our experiment lies between 1 and 10 .

The probability of observing $n_{1}$ individuals of $\mathrm{ST} / \mathrm{CH}$ is then:

$$
\begin{aligned}
\operatorname{pr}\left(n_{1} \mid \pi_{k}, n_{e}\right) & \\
= & \left(\begin{array}{c}
n \\
n_{1}
\end{array}\right) \frac{\Gamma\left(n_{e}\right) \Gamma\left(n_{1}+n_{e} \pi_{k}\right) \Gamma\left(n_{2}+n_{e} \bar{\pi}_{k}\right)}{\Gamma\left(n+n_{e}\right) \Gamma\left(n_{e} \pi_{k}\right) \Gamma\left(n_{e} \bar{\pi}_{k}\right)} .
\end{aligned}
$$

The model for one sample is then beta-binomial (Levin and Reeds, 1977, and Brier, 1980).

The salient feature of the model is that allowing the probabilities of survival to be random inflates the variance on the counts by a factor $\mathrm{C}$, so that $\operatorname{VAR}\left(n_{1}\right)=n C \pi_{k} \bar{\pi}_{k}$, and $\operatorname{COV}\left(n_{1}, n_{2}\right)=-n C \pi_{k} \bar{\pi}_{k}$. The constant $C$ is $\left(n+n_{e}\right) /\left(1+n_{e}\right)$, where the sample size $n$ is usually 10 , and $C$ is some number between 1 and 10 . The quantity $n / C$ may be considered the effective sample size, the number of 
Table 2 Analysis of variability among replicate samples, usually* of size 10: $\chi^{2}$, the chi-squared statistic of homogeneity; df $=a_{t}-1$ or $2 a_{t}-2$, its degrees of freedom; $P$, the proportion of ST/CH, used to compute expected frequencies; $\hat{C}_{t}$, the estimate of scale; and $\alpha$, the significance level of homogeneity tests

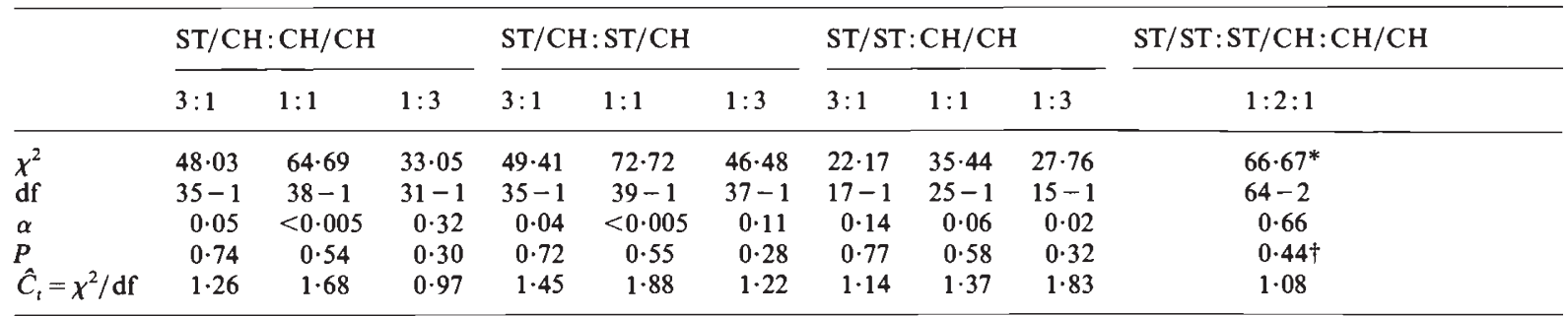

* The ordinary $\chi^{2}$ test of homogeneity is reported here over all replicates because sample size varied much more than in pairwise experiments.

† The proportion of ST/ST used was 0.349 from table 1 .

individuals necessary to produce the excess variability of a sample under binomial sampling only.

According to Theorem 1 in Levin and Reeds (1977), the likelihood has one global maximum with respect to the parameters in our model, and thus the method of maximum likelihood is a natural one. Since the results based on the method of maximum likelihood do not differ substantially from a simpler analysis suggested by Brier (1980), the simpler analysis is presented. As in Brier (1980), estimates of viabilities (or expected survival probabilities) can be obtained by proceeding as if the survival probabilities $\theta_{k}$ were fixed, but their variances are rescaled by $C$. The estimates and their standard errors (S.E.) are as follows:

$\hat{V}_{k}=k N_{2} /\left[1+N_{1}\right]$

S.E. $\hat{V}_{k}=\sqrt{\left[\hat{C} \hat{V}_{k}\left(k+\hat{V}_{k}\right)^{2} / N\right]}$;

$\hat{\pi}_{k}=N_{1} / N ;$ and S.E. $\hat{\pi}_{k}=\sqrt{\hat{C} \hat{\pi}_{k} \hat{\bar{\pi}}_{k} / N}$.

The count $N_{1}=\sum n_{1}$ (or $N_{2}=\Sigma n_{2}$ ) pools over samples at a given input ratio and in a given experiment, with $N=N_{1}+N_{2}$. Similar estimates can be derived for the experiment with three karyotypes. An estimate of the scale parameter $C$ (or equivalently $n_{e}$ ) is needed. The scale estimate $\hat{C}$ is also used to rescale chi-squared tests of fit to the null hypothesis, or to other models.

Brier (1980) suggests a natural estimate for the scale parameter $C$. Let us suppose that there are $a_{t}$ cultures of sample size $t$ in the first experiment (ST/CH vs. $\mathrm{CH} / \mathrm{CH}$ ). A chi-squared test for homogeneity between all cultures of size $t$ is then computed as

$$
\chi_{a_{t}-1}^{2}=\Sigma\left[\left(n_{1}-P t\right)^{2} / P t+\left(n_{2}-Q t\right)^{2} / Q t\right] \delta(n-t) .
$$

The indicator function $\delta$ sets aside those samples not of size $t$. The proportion $P=1-Q$ is the fraction of ST/CH adults in samples of size $t$ in the first experiment. Brier (1980) proposed estimating the scale parameter $C_{t}$ by $\hat{C}_{t}=\chi_{a_{t}-1}^{2} /\left(a_{t}-1\right)$, namely the chi-squared statistic divided by its degrees of freedom, $a_{t}-1$. For the experiment with three karyotypes, the degrees of freedom become $2 a_{t}-2$. The scale parameter estimate is then a natural measure of homogeneity among replicates. The parameter estimates above are fairly efficient relative to the method of maximum likelihood. In order to generate a pooled estimate of the scale parameter $C$ for an entire experiment, a weighted average of the scale estimates $\hat{C}_{t}$ from samples of different sizes is taken as $\hat{C}=\Sigma_{t} a_{t} t \hat{C}_{t} /\left(\Sigma_{t} a_{t} t\right)$. This scale estimate summarises the additional variability incurred by beta-binomial sampling.

Tests of homogeneity are reported in table 2 for each input ratio in each experiment. These tests indicate significant heterogeneity at two input ratios in pairwise experiments involving the heterokaryotype. An estimate of the scaling constant $\hat{C}$ measuring the variability at each input ratio is also reported (table 2 ) to allow us to compute standard errors on viability estimates, as in equation (2).

Any test of a hypothesis about the form of viability selection, i.e., whether or not the viabilities are frequency-dependent, must be done relative to the additional variability measured by the scaling constant $C$. For example, under the null hypothesis that selection is frequency-dependent, the expected frequencies are $m_{1}=n-m_{2}=\hat{\pi}_{k} n$. Goodness of fit to this hypothesis may be tested as

$$
\chi^{2}=\Sigma\left[\left(n_{1}-m_{1}\right)^{2} / m_{1}+\left(n_{2}-m_{2}\right)^{2} / m_{2}\right] .
$$

The sum is over samples in an experiment (e.g., table 3 , part 2), and the degrees of freedom $d f_{0}$ are 
Table 3 Tests of hypotheses about frequency-dependent selection: the estimated scale parameters $\hat{C}$ for each experiment; chi-squared test statistics; their degrees of freedom, df; significance levels $\alpha$ for nested hypotheses; and $D$, the difference in chi-squared statistics which measures improvement in fit between two hypotheses

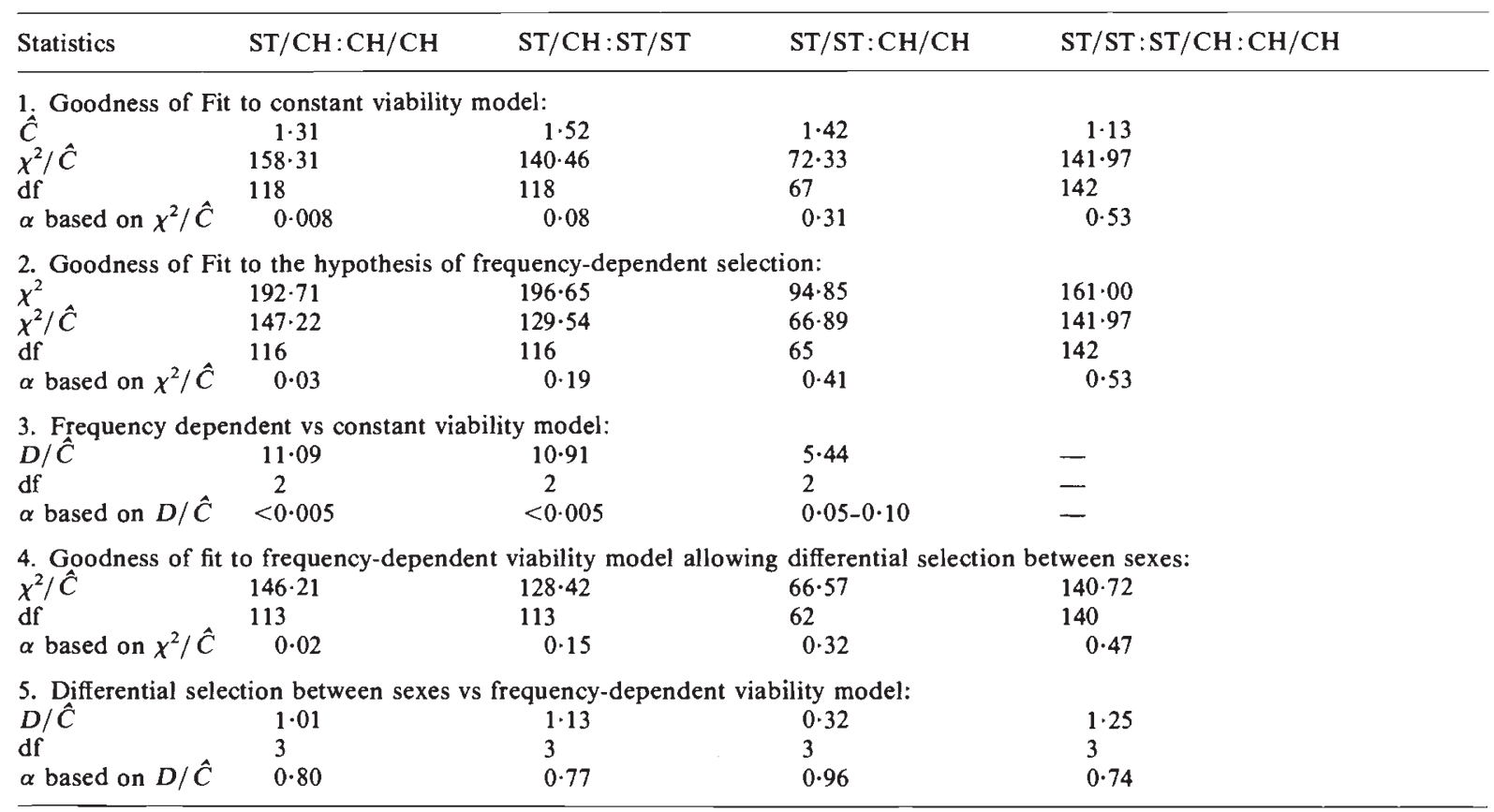

the number of samples in the experiment minus the number of viabilities estimated in the experiment. Brier (1980) shows that this statistic $\chi^{2} / \hat{C}$ has a chi-squared distribution with degrees of freedom $d f_{0}$ in large samples under the null hypothesis. Again, $\hat{C}$ enters as a scale factor.

As under the null hypothesis, estimates of viabilities under an alternative hypothesis can be obtained by proceeding as if survival probabilities were fixed. For some of the alternatives, estimates are computed iteratively. As an example, under the constant viability hypothesis there is a single viability $V$ for $\mathrm{CH} / \mathrm{CH}$. If the survival probabilities were constant as karyotypic input ratio is varied (e.g., $V_{k}=V$ for all $k$ ), then the likelihood for an experiment would be a product of independent binomial densities for each sample. Setting the loglikelihood's derivative to zero yields:

$$
\Sigma \frac{N_{1}}{k+V}=\Sigma \frac{k N_{2}}{V(k+V)},
$$

where $N_{1}=N-N_{2}$ is the count of ST/CH in all samples at input ratio $k$. The Newton-Raphson procedure can be used iteratively to solve equation (5). The resulting estimate $\hat{V}$ can be inserted into the equation $m_{1}=N-m_{2}=N \hat{\pi}=N k /(k+\hat{V})$ to obtain fitted counts in equation (4) under the constant viability hypothesis.
Hypotheses can be compared for goodness of fit by subtracting the goodness of fit statistic and degrees of freedom $\chi_{A}^{2} / \hat{C}\left(\mathrm{df}_{A}\right)$ of the hypothesis which has more parameters from the corresponding quantities $\chi^{2} / \hat{C}(\mathrm{df})$ of the hypothesis which has fewer parameters. This difference $D / \hat{C}=$ $\chi^{2} / \hat{C}-\chi_{A}^{2} / \hat{C}$ of chi-squared statistics has a chisquared distribution with degrees of freedom $(\mathrm{df}-$ $\mathrm{df}_{A}$ ), and it tests whether or not the more elaborate hypothesis allows a significant improvement over the more parsimonious one (e.g., table 3, part 3).

A contour plotting routine of Whitten (1973) was used to generate a graphical display of relative fitness as a function of karyotypic frequency. Isoclines of the fitness of each homokaryotype relative to the fitness of the heterokaryotype were plotted on a triangular coordinate system whose axes were the karyotypic frequencies, and the results are shown in fig. 1.

\section{RESULTS}

The first step in the analysis is to test the simplest hypothesis, that viability selection was constant from egg to adult. For example, the constant relative viability of two karyotypes was estimated from the total number of each karyotype $\left(N_{1}, N_{2}\right)$ sum- 
med over all samples at each input frequency within one experiment. These counts of karyotypes were then used to obtain a viability estimate with equation (5). This constant viability was then used to calculate expected frequencies of the two karyotypes in each sample according to $m_{1}=\hat{\pi}_{k} n$, and a chi-squared test for fit between observed and expected frequencies was performed as in equation (4). The results are given in table 3 (part 1). The constant viability model was rejected in competition experiments involving (ST/CH: $\mathrm{CH} / \mathrm{CH}$ ), was borderline in the experiment involving (ST/CH:ST/ST), and was acceptable in the pairwise competition experiment between the two homokaryotypes and in the three-way experiment (table 3, part 1). If the overall fit to the constant viability model is examined $\left(\chi^{2} / \hat{C}=158 \cdot 31+\right.$ $140 \cdot 46+72 \cdot 33+141.97=513 \cdot 07, \quad \mathrm{df}=445, \quad \alpha=$ 0.014 ), the model's fit is unacceptable at the 0.05 level.

The next simplest hypothesis allows selection to be frequency-dependent, but without epistatic interactions in fitness between karyotypes. Under this hypothesis, a genotype's fitness does not depend on which other karyotype is also present in the vial; for example, the relative viability of ST/ST in combination with ST/CH does not differ from its viability in combination with $\mathrm{CH} / \mathrm{CH}$. We now test for goodness of fit to this model without fitness interactions. Only six relative viabilities are necessary in this model, one for each homokaryotype at ratios of $3: 1 ; 1: 1$; and $1: 3$. The fitnesses of the karyotypes in the three-way mixture were predicted by linear interpolation from the pairwise experiments to be $1.03: 1: 0.88$ for $\mathrm{ST} / \mathrm{ST}: \mathrm{ST} / \mathrm{CH}: \mathrm{CH} / \mathrm{CH}$. These values may be contrasted to those of table 1 , line 10 , where viabilities were estimated by Haldane's (1956) formulae. These estimates were then used to compute expected frequencies in a goodness of fit test to the hypothesis of no interaction in fitness, by means of equation (4). The test of fit was performed using a global estimate of the scaling constant over all four experiments, $\hat{C}=1 \cdot 362$, yielding $\chi^{2} / \hat{C}=$ $647 \cdot 82 / 1 \cdot 362=475 \cdot 64$, with $\mathrm{df}=441$ and $\alpha<$ $0 \cdot 001$. We conclude that there is a significant interaction between karyotypes and that we are justified in analysing each experiment separately. A clear contribution to the interaction comes from a predicted fitness of 1.03 for ST/ST with no epistasis, when in fact it is estimated as 1.58 in the experiment involving three karyotypes (table 1).

Next we test the goodness of fit to the hypothesis that the viability selection is frequency dependent and epistatic. The relative viability of two karyotypes in a particular combination of karyotype and frequency was estimated from the total number of each karyotype summed over all samples by equation (2). These viabilities were then used to calculate the expected frequencies of karyotypes in each sample according to equation (2) again, and a chi-squared test for fit between observed and expected frequencies was performed as in equation (4). Fit to the hypothesis that selection changed with frequency was acceptable (table 3 , part 2), although there was an excess of variability in the competition experiment involving ST/CH and $\mathrm{CH} / \mathrm{CH}$ which resulted in an $\alpha$ of 0.03 . The individual tests of goodness of fit can be combined $\left\{\chi^{2} \hat{C}=147 \cdot 22+129 \cdot 54+66 \cdot 89+141 \cdot 97=485 \cdot 62\right.$, $\mathrm{df}=439, \alpha=0.066\}$, indicating that the null hypothesis is acceptable. The residuals $\hat{e}=$ $(n-m) / \sqrt{m}$ were plotted in various ways (Snedecor and Cochran, 1980): (i) normal plots of $\hat{e}$ 's, (ii) $\hat{e}$ vs. $k$, and (iii) $\hat{e}$ vs. karyotype. They fitted a normal distribution well by various criteria (e.g., Anscombe and Glynn, 1983) and showed no pattern of variation with different combinations of karyotypes or with the input ratios of the karyotypes. The residual plots, then, revealed no pattern of departure from our hypothesis that viabilities are frequency-dependent and epistatic. Furthermore, comparing the goodness of fit of the frequency-dependent selection hypothesis with the constant viability hypothesis (table 3 , part 3 ), we see that the two tests for frequency-dependent selection involving the heterokaryotype are significant, but the test involving only the homokaryotypes is not.

Dobzhansky and Levene (1948) demonstrated the possibility of differential selection between the sexes in natural populations, although this effect has not been reported in laboratory experiments on the third chromosome polymorphism in D. pseudoobscura. Viability estimates were computed for each sex and input ratio by analogy to equation (2), permitting the construction of the chi-squared tests with equation (4). The fit was reasonable (table 3 , part 4 ) but not a significant improvement (table 3, part 5) over the null hypothesis without differential selection between the sexes. We conclude that the more parsimonious hypothesis of frequency-dependent selection with viability the same in males and females explains our experiments better.

The heterogeneity between samples is in excess of that predicted by binomial sampling (table 2), as also found by other experimenters (Prout, 1971 and Clark and Doane, 1984). The estimates of the scale parameter in table 3 indicate a 30 to 50 per 
cent increase in variability over that arising under binomial sampling alone in the pairwise experiments $(\hat{C}=1 \cdot 31)$. This variability translates into an effective sample size of $n / \hat{C}=7$ or 8 individuals of each sex from each vial in the pairwise experiments, in contrast to the actual sample size of 10 . Thus, the standard errors of viabilities are affected substantially by the variability between samples.

Finally, the hypothesis of constant viability was compared directly with the hypothesis of frequency dependent selection (table 3, part 3). The constant viability model was rejected in competition experiments involving the heterokaryotype, but was acceptable in the pairwise competition experiment between homokaryotypes. The chi-squared tests (table 3) globally summarise the departures from the constant viability hypothesis, but do not indicate how this hypothesis fails to fit the data. It is instructive to compare viabilities at different input ratios, although these individual comparisons will be more limited in their ability to detect departures from the constant viability hypothesis. These comparisons are made with the understanding that any particular comparison will appear to be less significant than the global test, since an individual comparison is not based on all the data. All of the comparisons must be taken together to explain the failure of the constant viability hypothesis.

The most instructive comparisons are between the relative viabilities of the same homokaryotype at different frequencies. The relative viability of either ST/ST or $\mathrm{CH} / \mathrm{CH}$ was higher at the low (1/4) homokaryotypic frequency than at the high (3/4) frequency. We can use the estimates of viability and their standard errors to calculate the usual normal deviate $Z=\left(\hat{V}_{1}-\hat{V}_{2}\right) /$ S.E. $\left(\hat{V}_{1}-\hat{V}_{2}\right)$. For $\mathrm{ST} / \mathrm{ST}$, this comparison gave $Z=1 \cdot 86$, with a probability $(\alpha)$ of 0.06 and $\hat{C}=1.309$; for $\mathrm{CH} / \mathrm{CH}$, this comparison gave $Z=1.84$, with $\alpha=0.06$ and $\hat{C}=1 \cdot 518$.

Comparisons between the relative viabilities of different karyotypes are also informative. At the lowest homokaryotypic frequency (1/4), the viabilities of the homokaryotypes were not demonstrably different from that of the heterokaryotype; that is, the relative viabilities did not differ significantly from unity. These results are demonstrated most easily by forming approximate 95 per cent confidence intervals about the relative viabilities as twice their standard errors, and noting that the intervals overlap 1. Similarly, we see that at higher homokaryotypic frequencies $(1 / 2$ and $3 / 4)$ the viabilities of the homokaryotypes were significantly lower than that of the heterokaryotype.
The two homokaryotypes were also compared at three relative frequencies. The viability of $\mathrm{CH} / \mathrm{CH}$ relative to that of ST/ST was not significantly different when $\mathrm{CH} / \mathrm{CH}$ frequency was $1 / 4$ than when it was $3 / 4$; for this comparison $Z=1 \cdot 39$ and $\alpha=0 \cdot 16$. There was thus no clear evidence for a frequency-dependent advantage of one homokaryotype over the other. There was, however, an indication that ST/ST was somewhat more viable than $\mathrm{CH} / \mathrm{CH}$. When the homokaryotypes were paired at equal frequencies, or when the frequency of $\mathrm{CH} / \mathrm{CH}$ was $3 / 4, \mathrm{ST} / \mathrm{ST}$ was significantly more viable than $\mathrm{CH} / \mathrm{CH}$. In addition, for each of the three frequencies at which a homokaryotype was paired with the heterokaryotype, the viability of $\mathrm{CH} / \mathrm{CH}$ relative to that of ST/CH was estimated to be lower than the corresponding viability of ST/ST measured relative to that of ST/CH; none of these differences was statistically significant, however. Lastly, when all three karyotypes were competed together, no heterozygote advantage was demonstrable, and again the viability of $\mathrm{CH} / \mathrm{CH}$ relative to that of $\mathrm{ST} / \mathrm{CH}$ was significantly lower than the corresponding viability of ST/ST measured relative to that of ST/CH. Fig. 1 summarises the response of homokaryotypic relative fitness as a function of input frequency of karyotypes and shows that as either homokaryotype becomes rare, its relative fitness increases.

\section{DISCUSSION}

The hypothesis of frequency-dependent selection with a random component due to small microenvironmental differences between replicates accounts for our data better than the alternative of constant selection, and this model differs from earlier ones in providing an adequate explanation of variability between replicates. The viabilities we have measured do not show a consistent heterozygous advantage when we extrapolate from experiments on pairs of karyotypes or when all three karyotypes are put together. Estimated viabilities of the ST/ST and $\mathrm{CH} / \mathrm{CH}$ homokaryotypes are not significantly different from that of the ST/CH heterokaryotype when the ratio of homokaryotypes to heterokaryotypes is $1: 3$. The heterokaryotype does have a significantly higher viability than either homokaryotype when the frequencies of homokaryotypes and heterokaryotypes are each $1 / 2$. At such frequencies, the other homokaryotypes would be at low frequency, and our measurements indicate that its viability would not differ 
from that of the heterokaryotype. Thus the heterozygote advantage which we found at equal frequency of each homokaryotype and the heterokaryotype would be one-sided, with the more frequent homokaryotype having a lower viability than the heterokaryotype, while the other, less frequent homokaryotype would have a viability at least equal to that of the heterokaryotype.

The consequences of such viability selection can be seen by noting that the heterokaryotype was favoured whenever the frequency of a homokaryotype was $3 / 4$ and that of the heterokaryotype 1/4. The other homokaryotype was quite rare and, according to our measurements, did not differ from the heterokaryotype in viability, or even enjoyed an advantage. At such frequencies the heterokaryotype and the rare homokaryotype would have a higher viability than the more frequent homokaryotype. There would be no heterozygote advantage, but the result would be selection which increases the frequency of the rare gene arrangement. The polymorphism would be protected, and selection would favour either gene arrangement should it become rare. The nature of the viabilities which underly this protected polymorphism are graphically displayed in fig. 1 .

It is clear from our study of viability, and from the comparable study of fertility by Anderson and Watanabe (1974), that the polymorphism for gene arrangements in D.pseudoobscura is not necessarily a case of simple heterozygote advantage, but may well involve frequency-dependent selection. In fact, our measurements of viability do not indicate heterozygote advantage in breeding populations with the three karyotypes present.

Viability is not, of course, the only component of fitness. Selection also operates through differences in various aspects of reproduction, such as female fecundity and male mating success. Anderson and Watanabe (1974) found large fertility differences among ST/ST, ST/AR, and AR/AR karyotypes derived from the same sample of the population at Mather. This fertility selection varied with karyotypic frequency in a way that would contribute to a balancing selection on the gene arrangement. Anderson and Brown (1984) studied male mating success in experimental populations containing the same $\mathrm{ST} / \mathrm{ST}, \mathrm{ST} / \mathrm{CH}$, and $\mathrm{CH} / \mathrm{CH}$ karyotypes used in this study. They found a significant mating advantage of rare male homokaryotypes, relative to the mating success of the heterokaryotypes. The heterokaryotypes did not show a rare male advantage. This pattern of mating advantage for rare male homokaryotypes would contribute to balancing selection, although it is not itself sufficient to bring about genetic equilibrium.

Dobzhansky and Levene (1948) showed that selection by differential viability occurred on the $D$. pseudoobscura karyotypes in nature, and Anderson et al. (1979) and Salceda and Anderson (1985) showed that differential fertility has an important role in nature. Thus, both viability and fertility components of fitness seem to be important in balancing selection on this chromosomal polymorphism in $D$. pseudoobscura. The relative role of these two components of fitness differ from organism to organism, and probably from gene to gene within an organism. In some cases, viability dominates (e.g., Anderson, 1969, and Clegg and Allard, 1973); in some fertility is more important (e.g., Bundgaard and Christiansen, 1972, and Clark and Feldman (1981)); and in others (e.g., Polivanov and Anderson, 1969), as with the D. pseudoobscura gene arrangements, both have major effects. Unfortunately, our measurements of the two components in Drosophila are for different sets of karyotypes, and in separate experiments. The relative roles of viability and fertility selection on $D$. pseudoobscura karyotypes within a single population are the subject of a current experiment.

The general subject of frequency-dependent selection has been reviewed by Petit and Ehrman (1969) and Ayala and Campbell (1974), and these articles as well as the recent research reports of Snyder and Ayala (1979), Tosic and Ayala (1981), and Nunney (1983), may be consulted for references to the large literature on this topic. Frequencydependent viabilities of the sort we found, where the fitness of a genotype is greater at low genotypic frequencies, have been reported for other Drosophila species. Frequency-dependence of fitness, and especially the "minority advantage" form of it, seems to be a general phenomenon and may even turn out to be more the rule than the exception. Gromko (1977) has gone so far as to propose that the term frequency-dependent selection be restricted to balancing selection in which genotypes have higher fitness as they become rarer and depart further from equilibrium frequencies. We prefer to keep a broader definition of frequency-dependent selection which includes any regular change of fitness with gene or genotype frequency, while recognising the particular importance of the "minority advantage" form.

Acknowledgements We thank Ms Margaret Elaine Little and Mrs Carol A. Yardley for technical assistance in the experiments, and Celeste Brown, Jørgen Bundgaard, Andrew Clark, 
Timothy Prout, Vibeke Simonsen and Alan Wilton for helpful discussions of our analysis. This research was supported by the U.S. National Science Foundation under Grants DEB-7918493 and BSR-831582.

\section{REFERENCES}

ANDERSON, w. W. 1969. Selection in experimental populations. I. Lethal genes. Genetics, 62, 653-672.

ANDERSON, W. W. AND BROWN, C. J. 1984. A test for rare male mating advantage with Drosophila pseudoobscura karyotypes. Genetics, 107, 577-589.

ANDERSON, W. W., LEVINE, L., OLVERA, O., POWELL, J. R., DE LA ROSA, M. E., SALCEDA, V. M., GASO, M. I. AND GUZMAN, J. 1979. Evidence for selection by male mating success in natural populations of Drosophila pseudoobscura. Proc. Nat. Acad. Sci. USA, 76, 1519-1523.

ANDERSON, W. W., OSHIMA, C., WATANABE, T., DOBZHANSKY, TH. AND PAVLOVSKY, O. 1968. Genetics of natural populations. XXXIX. A test of the possible influence of two insecticides on the chromosomal polymorphism in Drosophila pseudoobscura. Genetics, 58, 423434.

ANDERSON, w. W. AND watanabe, T. K. 1974. Selection by fertility in Drosophila pseudoobscura. Genetics, 77, 559-564.

ANSCOMBE, F. J. AND GLYNN, W. J. 1983. Distribution of the kurtosis $b_{2}$ for normal samples. Biometrika 70, 227-234.

ARNOLD, J. 1981. Statistics of natural populations. 1: Estimating an allele probability in cryptic fathers with a fixed number of offspring. Biometrics, 37, 495-504.

ARNOLD, J. AND MORRISON, M. L. 1985. Statistics of natural populations. II: Estimating an allele probability in families descended from cryptic mothers. Genetics 109, 785-798.

AYALA, F. J. AND CAMPBELL, C. A. 1974. Frequency-dependent selection. Annual Rev. Ecol. Syst., 5, 115-138.

BRIER, S. S. 1980. Analysis of contingency tables under cluster sampling. Biometrika 67, 591-596.

BUNDGAARD, J. AND CHRISTIANSEN, F. B. 1972. Dynamics of polymorphisms: I. Selection components in an experimental population of Drosophila malanogaster. Genetics, $71,439-460$.

CLARK, A. G. AND DOANE, w. W. 1984. Interactions between the amylase and adipose chromosomal regions of Drosophila melanogaster. Evolution, 38, 957-982.

CLARK, A. G. AND FELDMAN, M. W. 1981. Density-dependent fertility selection in experimental populations of Drosophila melanogaster. Genetics, 98, 849-869.

CLARK, B. C. 1962. Balanced polymorphism and diversity of sympatric species. In Nichols, D. (ed.) Taxonomy and Geography, Systematics Association Publication No. 4.

CLEGG, M. T. AND ALLARD, R. W. 1973. Viability versus fecundity selection in the slender wild oat, Avena barbata $L$. Science, 181, 667-668.

CORNELL, J. 1973. Experiments with mixtures: a review. Technometrics, 15, 437-455.

CORNELL, J. 1979. Experiments with mixtures: an update and bibliography. Technometrics, 21, 95-106.

DAWOOD, M. M. AND STRICKBERGER, M. W. 1969. The effect of larval interaction on viability in Drosophila melanogaster. III. Effects of biotic residues. Genetics, 63, 213-220.

DOBZHANSKY, TH. 1943. Genetics of natural populations. IX. Temporal changes in the composition of populations of Drosophila pseudoobscura. Genetics, 28, 162-186.
DOBZHANSKY, TH. 1944. Chromosomal races in Drosophila pseudoobscura and its relatives. In Dobzhansky, Th. and Epling, C. Contributions to the Genetics, Taxonomy, and Ecology of Drosophila Pseudoobscura and Its Relatives, Carnegie Institution of Washington Publication 554.

DOBZHANSKY, TH. 1947a. Adaptive changes induced by natural selection in wild populations of Drosophila. Evolution, $1,1-16$.

DOBZHANSKY, TH. $1947 \mathrm{~b}$. Genetics of natural populations. XIV. A response of certain gene arrangements in the third chromosome of Drosophila pseudoobscura to natural selection. Genetics, 32, 142-160.

DOBZHANSKY, TH. 1951. Genetics and the Origin of Species, Third Edition. Columbia University Press, New York.

DOBZHANSKY, TH. 1970. Genetics of the Evolutionary Process. Columbia University Press, New York.

DOBZHANSKY, TH., AYALA, F. J., STEBBINS, G. L. AND VALEN. TINE, J. W. 1977. Evolution. W. H. Freeman, San Francisco.

DOBZHANSKY, TH. AND LEVENE, H. 1948. Genetics of natural populations. XVII. Proof of operation of natural selection in wild populations of Drosophila pseudoobscura. Genetics, $33,537-547$.

EHRMAN, L., SPASSKY, B., PAVLOVSKY, O. AND DOBZHANSKY, TH. 1965. Sexual selection, geotaxis, and chromosomal polymorphism in experimental populations of Drosophila pseudoobscura. Evolution, 19, 337-346.

FIENBERG, S. E. 1980. The Analysis of Cross-Classified Categorical Data, 2nd Edition, MIT Press, Cambridge.

FISHER, R. A. 1922. On the dominance ratio. Proc. Roy. Soc. Edinburgh, 42, 321-341.

GROMKO, M. H. 1977. What is frequency-dependent selection? Evolution, 31, 438-442.

HALDANE, J. B. S. 1924. A mathematical theory of natural and artificial selection. Part 1. Trans. Cambridge Phil. Soc., 23, 19-41.

HALDANE, J. B. S. 1956. The estimation of viabilities. J. Genetics, $54,294-296$.

LEVENE, H. 1953. Genetic equilibrium when more than one ecological niche is available. Amer. Nat., 87, 331-333.

LEVENE, H., PAVLOVSKY, O. AND DOBZHANSKY, TH. 1954. Interaction of the adaptive values in polymorphic experimental populations of Drosophila pseudoobscura. Evolution, 8, 335-349.

LEVIN, B. AND REEDS, J. 1977. Compound multinomial likelihood functions are unimodal: proof of a conjecture of I. J. Good, Ann. of Statist., 5, 79-87.

LEWONTIN, R. C. 1955. The effects of population density and composition on viability in Drosophila melanogaster. Evolution, 9, 27-41.

NUNNEY, L. 1983. Sex differences in larval competition in Drosophila melanogaster: the testing of a competition model and its relevance to frequency-dependent selection. Amer. Nat., 121, 67-93.

PETIT, C. AND EHRMAN, L. 1969. Sexual selection in Drosophila. In Dobzhansky, Th., Hecht, M. K. and Steere, W. C. (eds.), Evolutionary Biology, 7, Plenum Press, New York.

POlivanOV, S. AND ANDERSON, w. w. 1969. Selection in experimental populations: II. Components of selection and their fluctuations in two populations of Drosophila melanogaster. Genetics, 63, 919-932.

PROUT, T. 1968. Sufficient conditions for multiple niche polymorphism. Amer. Nat., 102, 493-496.

PROUT, T. 1971. The relation between fitness components and population prediction in Drosophila. I: The estimation of fitness components. Genetics 68, 127-149. 
SALCEDA, V. M. AND ANDERSON, W. W. 1985. Rare male mating advantage in a natural population of Drosophila. Proc. Natl. Acad. Sci. USA (in press).

SNEDECOR, G, W. AND COCHRAN, W. G. 1980. Statistical Methods, 7th Edition. Iowa State University Press, Ames.

SNYDER, T. P. AND AYALA, F. J. 1979. Frequency-dependent selection at the PGM-1 locus of Drosophila pseudoobscura. Genetics, 92, 995-1003.

TOSIC, M. AND AYALA, F. J. 1981. Density and frequencydependent selection at the $M d h-2$ locus in Drosophila pseudoobscura. Genetics, 97, 679-701.

WHITTEN, T. 1973. Orthogonal-polynomial contoured trendsurface maps for irregularly-spaced data, Comp. App., 1, 171-192.
WRIGHT, S. 1931. Evolution in Mendelian populations. Genetics, 16, 97-159.

WRIGHT, S. AND DOBZHANSKY, TH. 1946. Genetics of natural populations. XII. Experimental reproduction of some of the changes caused by natural selection in certain populations of Drosophila pseudoobscura. Genetics, 31, 125-156.

YARDLEY, D. G., ANDERSON, W. W. AND SCHAFFER, H. E. 1977. Gene frequency changes at the $\alpha$-amylase locus in experimental populations of Drosophila pseudoobscura. Genetics, 87, 357-369.

YOSHIMARU, H. AND MUKAI, T. 1979. Lack of experimental evidence for frequency-dependent selection at the alcohol dehydrogenase locus in Drosophila melanogaster. Proc. Natl. Acad. Sci. USA, 76, 876-878. 